\title{
BMI-Based Neurocontroller for State-Feedback Guaranteed Cost Control of Discrete-Time Uncertain System
}

\author{
Hiroaki Mukaidani \\ Graduate School of Education \\ Hiroshima University \\ 1-1-1 Kagamiyama, Higashi-Hiroshima \\ 739-8524 Japan. \\ Email: mukaida@hiroshima-u.ac.jp
}

\author{
Seishiro Sakaguchi, Yasuhisa Ishii and Toshio Tsuji \\ Graduate School of Engineering \\ Hiroshima University \\ 1-4-1 Kagamiyama, Higashi-Hiroshima \\ 739-8527 Japan. \\ Email: \{sakaguchi, y_ishii, tsuji\}@bsys.hiroshima-u.ac.jp
}

\begin{abstract}
The application of neural networks to the statefeedback guaranteed cost control problem of discrete-time system that has uncertainty in both state and input matrix is investigated. Based on the Bilinear Matrix Inequality (BMI) design, a class of a state feedback controller is newly established, and sufficient conditions for the existence of guaranteed cost controller are derived. The novel contribution is that the neurocontroller is substituted for the additive gain perturbations. It is newly shown that although the neurocontroller is included in the discrete-time uncertain system, the robust stability for the closed-loop system and the reduction of the cost are attained.
\end{abstract}

\section{INTRODUCTION}

In recent years, the guaranteed cost control problem for a class of the uncertain discrete-time system which is based on the LMI design approach has been widely studied in the literatures (See e.g., [1], [2], [3]). The guaranteed cost control approach has the advantage of providing an upper bound on a given performance index. However, due to the presence of the design parameter of the LMI conditions, it is well-known that the cost becomes quite large.

In recent years, Bilinear Matrix Inequality (BMI) formulation of the control problems was made popular. Although it seems to be hard to solve the BMI, there were several attempts to solve BMI problems numerically that is based on branchand-bound schemes [4]. As a result, it is possible to tackle the BMI problems by applying such non-linear optimization techniques.

A neural network (NN) has been actively exploited to construct an intelligent control system because of its nonlinear mapping approximation for the system uncertainties involved. Then some control methodologies utilizing $\mathrm{NN}$ have been proposed by combining the modern control theory. For example, the Linear Quadratic Regulator (LQR) problem using the multiple NN has been investigated [5]. However, in these researches, there is a possibility that the existing neurocontroller may not stabilize the plant because the stability of the closed-loop system which includes the neurocontroller has not been considered. In fact, it has been shown that the system stability is destroyed when the degree of system nonlinearity is strong [5]. In order to avoid this problem, the stability of the closed-loop system with the neurocontroller has been studied via the LMI-based design approach [8], [9]. However, in these researches, the uncertainty in the input matrix has not been considered.

In this paper, the guaranteed cost control problem of the discrete-time uncertain system that has uncertainty in both state and input matrices is discussed. This is an extension of the former results [8], [9] in the sense that the uncertainty in the input matrix is included. A class of the fixed state feedback controller of the discrete-time uncertain system with the gain perturbations is newly established by means of the Bilinear Matrix Inequality (BMI). A new idea is that the neurocontroller is substituted for the additive gain perturbations. As a result, although the neurocontroller is included in the discretetime uncertain system, the robust stability of the closed-loop system and the reduction of the cost are attained.

\section{PRELIMINARY}

Consider the following class of an uncertain discrete-time linear system:

$$
\begin{aligned}
x(k+1)= & {\left[A+D F(k) E_{a}\right] x(k) } \\
& +\left[B+D F(k) E_{b}\right] u(k), x(0)=x^{0}, \\
u(k)= & {\left[K+D_{k} N(k) E_{k}\right] x(k), }
\end{aligned}
$$

where $x(k) \in \Re^{n}$ is the state, $u(k) \in \Re^{m}$ is the control input, $A, B, D, D_{k}, E_{a}, E_{b}$ and $E_{k}$ are known constant matrices, $K$ is the fixed control matrix for the controller (1b), and $F(k) \in$ $\Re^{p_{a} \times q_{a}}$ is unknown matrix function and $N(k) \in \Re^{p_{n} \times q_{n}}$ is the output of NN. It is assumed that $F(k)$ and $N(k)$ satisfy

$$
F^{T}(k) F(k) \leq I_{q_{a}}, N^{T}(k) N(k) \leq I_{q_{n}} .
$$

The block diagram of the new proposed method is shown in Fig. 1, where $L$ is a time lag diagram. It should be noted that the controller (1b) has the neurocontroller as the additive gain perturbations $D_{k} N(k) E_{k}$ compared to existing method [5]. 


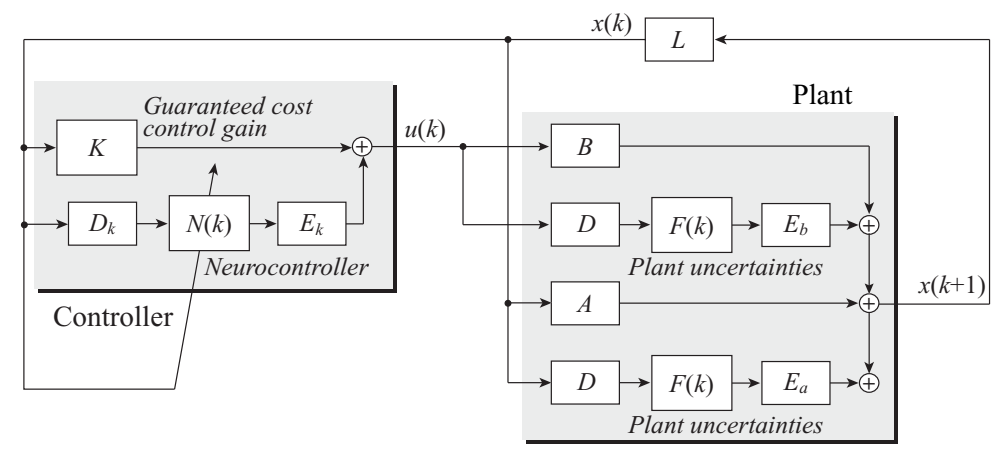

Fig. 1. Block diagram of a new proposed method.

Associated with the system (1) is the quadratic cost function

$$
J=\sum_{k=0}^{\infty}\left[x^{T}(k) Q x(k)+u^{T}(k) R u(k)\right],
$$

where $Q$ and $R$ are given as the positive definite symmetric matrices. In this situation, the definition of the guaranteed cost control with the additive gain perturbations is given below.

Definition 1: For the discrete-time uncertain system (1) and the cost function (3), if there exist a control matrix $K$ and a positive scalar $J^{*}$ such that for the admissible uncertainties and the neurocontroller (2), the closed-loop system is asymptotically stable and the closed-loop value of the cost function (3) satisfies $J<J^{*}$, then $J^{*}$ and $K$ are said to be the guaranteed cost and the guaranteed cost control matrix, respectively.

The following result shows that the guaranteed cost control for the system (1) has the upper bound on the cost function (3).

Lemma 1: Suppose that the following matrix inequality holds for the uncertain discrete-time system (1) with the cost function (3) and for all $x(k) \neq 0$.

$$
\begin{array}{r}
x^{T}(k+1) P x(k+1)-x^{T}(k) P x(k) \\
+x^{T}(k)\left[Q+\tilde{K}^{T} R \tilde{K}\right] x(k)<0,
\end{array}
$$

where $\tilde{K}:=K+D_{k} N(k) E_{k}$.

If such condition is met, the matrix $K$ of the controller (1b) is the guaranteed cost control matrix associated with the cost function (3). That is, the closed-loop uncertain system

$$
\begin{aligned}
& x(k+1) \\
= & {\left[\left(A+D F(k) E_{a}\right)\right.} \\
& \left.+\left(B+D F(k) E_{b}\right) \cdot\left(K+D_{k} N(k) E_{k}\right)\right] x(k),
\end{aligned}
$$

is stable and achieves

$$
J<J^{*}=x^{T}(0) P x(0) .
$$

Proof: Let us define the following Lyapunov function candidate

$$
V(x(k))=x^{T}(k) P x(k),
$$

where $P$ is the positive definite matrix. Since this proof can be done by using the similar technique in [8], it is omitted.

Theorem 1: Consider the uncertain discrete-time system (1) and cost function (3). For the uncertain matrix $F(k)$ and the gain perturbation matrix $N(k)$, if the BMI (8) has a feasible solution such as symmetric positive definite matrix $X \in \Re^{n \times n}$ and $Y \in \Re^{m \times n}$, and positive scalar $\mu>0$, then $K=Y X^{-1}$ is the guaranteed cost control gain matrix.

Furthermore, for all admissible uncertainties $F(k)$, and the gain perturbations $N(k)$, the corresponding value of the cost function (3) satisfies the following inequality (12)

$$
J<J^{*}=x^{T}(0) X^{-1} x(0) .
$$

Proof: Let us introduce the matrices $X=P^{-1}$ and $Y=$ $K P^{-1}$. Pre- and post-multiplying both sides of the inequality (8) by the positive definite matrix

$$
\text { block diag }\left(\begin{array}{lllllll}
P & I_{n} & I_{m} & I_{p_{n}} & I_{q_{a}} & I_{p_{a}} & I_{n}
\end{array}\right)>0
$$

and applying the Schur complement [7] gives (9). It should be noted that the BMI (8) is equivalent to the matrix inequality (9). Using a standard matrix inequality [6], for all admissible uncertainty, the matrix inequality (10) holds. Moreover, using a standard matrix inequality [6], for all the neurocontroller (2), the matrix inequality (10) results in (11). Finally, it is easy to verify that the matrix inequality (4) is satisfied. Thus, $K$ is the guaranteed cost control gain matrix. On the other hand, since the results of the cost bound (12) can be proved by using the similar argument for the proof of Theorem 1, it is omitted.

Since the BMI (8) consists of a solution set of $(\mu, X, Y)$, the efficient optimization algorithm such as branch and bound algorithm can be applied. Moreover, its solutions represent a set of the guaranteed cost control gain matrix $K$. This parameterized representation can be exploited to design the guaranteed cost control gain which minimizes the value of the guaranteed cost for the closed-loop uncertain system. Consequently, solving the following optimization problem allows us to determine the optimal bound.

$$
J<J^{*}<\min _{(\mu, X, Y)} \alpha,
$$

such that (8) and

$$
\left[\begin{array}{cc}
-\alpha & x^{T}(0) \\
x(0) & -X
\end{array}\right]<0 .
$$

The crucial difference between the uncertain discrete-time system in [1] and the considered system of this paper is that the controller gain perturbations as the neurocontroller are newly added. Furthermore, the BMI approach has been newly applied 


$$
\begin{aligned}
& {\left[\begin{array}{cccc}
-X & (*) & (*) & (*) \\
A X+B Y & -X & (*) & (*) \\
Y+\mu\left(E_{b} D_{k} D_{k}^{T}\right)^{T}\left(E_{a} X+E_{b} Y\right) & \left(B D_{k} D_{k}^{T}\right)^{T} & -R^{-1}+D_{k} D_{k}^{T}+\mu\left(E_{b} D_{k} D_{k}^{T}\right)^{T} E_{b} D_{k} D_{k}^{T} & (*) \\
\mu\left(E_{b} D_{k}\right)^{T}\left(E_{a} X+E_{b} Y\right) & \left(B D_{k}\right)^{T} & \mu\left(E_{b} D_{k}\right)^{T} E_{b} D_{k} D_{k}^{T} & -I_{p_{n}}+\mu\left(E_{b} D_{k}\right)^{T} E_{b} D_{k} \\
\mu\left(E_{a} X+E_{b} Y\right) & 0 & 0 & 0 \\
0 & D^{T} & 0 & 0 \\
X & 0 & 0 & 0
\end{array}\right.} \\
& \left.\begin{array}{ccc}
(*) & (*) & (*) \\
(*) & (*) & (*) \\
(*) & (*) & (*) \\
(*) & (*) & (*) \\
-\mu I_{q_{a}} & (*) & (*) \\
0 & -\mu I_{p_{a}} & (*) \\
0 & 0 & -\left(Q+E_{k}^{T} E_{k}\right)^{-1}
\end{array}\right]<0 \\
& {\left[\begin{array}{ccccc}
-P+Q+E_{k}^{T} E_{k} & (*) & (*) & (*) & (*) \\
A+B K & -P^{-1} & (*) & (*) & (*) \\
K+\mu\left(E_{b} D_{k} D_{k}^{T}\right)^{T}\left(E_{a}+E_{b} K\right) & \left(B D_{k} D_{k}^{T}\right)^{T} & -R^{-1}+D_{k} D_{k}^{T}+\mu\left(E_{b} D_{k} D_{k}^{T}\right)^{T} E_{b} D_{k} D_{k}^{T} & (*) & (*) \\
\mu\left(E_{b} D_{k}\right)^{T}\left(E_{a}+E_{b} K\right) & \left(B D_{k}\right)^{T} & \mu\left(E_{b} D_{k}\right)^{T} E_{b} D_{k} D_{k}^{T} & -I_{p_{n}}+\mu\left(E_{b} D_{k}\right)^{T} E_{b} D_{k} & (*) \\
E_{a}+E_{b} K & 0 & 0 & 0 & \mu^{-1} I_{q_{a}}
\end{array}\right]} \\
& =M<0 \text {. }
\end{aligned}
$$

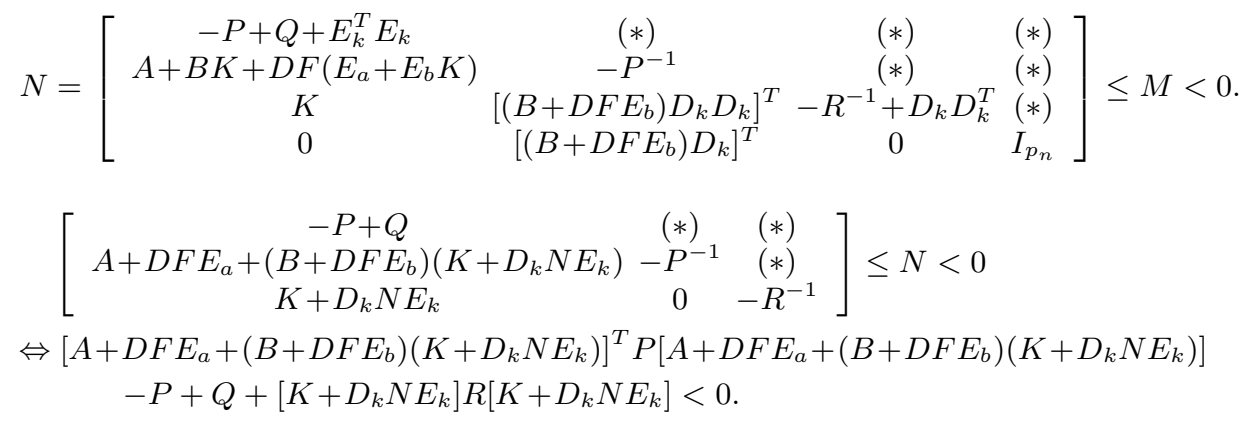

where the symbol $(*)$ in BMI denotes entries that follow from symmetry.

to the guaranteed cost control problem for the discrete-time system that includes the uncertainty in both state and control matrices compared to existing results [8], [9].

\section{NEURAL NETWORKS FOR ADDITIVE GAIN PERTURBATIONS}

The main purpose of this paper is to introduce $\mathrm{NN}$ as additive gain perturbations into the discrete-time uncertain system to improve the cost performance. It should be noted that the proposed neurocontroller regulates its outputs in real-time under the robust stability guaranteed by the BMI approach.

\section{A. On-line learning algorithm of neurocontroller}

It is expected that the reduction of the cost will be attained when the neurocontroller can manage the uncertain system as the nominal linear system while compensating for control errors by the conservative controller. That is, the neurocontroller is required to compensate the conservative controller to work as the LQR controller in the uncertain system.

Let us consider the following nominal system without the uncertainty and the gain perturbation.

$$
\hat{x}(k+1)=A \hat{x}(k)+B \hat{u}(k),
$$

where $\hat{x}(k) \in \Re^{n}$ is the state and $\hat{u}(k) \in \Re^{m}$ is the control input. For such linear system, it is well-known that the LQR control is an effective method to design the controller which can minimize the cost function (3). Based on the LQR, the optimal control $\hat{u}^{*}(k)$ can be designed as

$$
\begin{aligned}
\hat{u}^{*}(k) & =\hat{K} \hat{x}(k), \\
\hat{K} & =-\left(R+B^{T} \hat{P} B\right)^{-1} B^{T} \hat{P} A,
\end{aligned}
$$

where $\hat{K}$ is the optimal feedback gain matrix and the matrix $\hat{P}$ is the positive semidefinite symmetric solution of the following algebraic Riccati equation.

$$
\hat{P}=A^{T} \hat{P} A-A^{T} \hat{P} B\left(R+B^{T} \hat{P} B\right)^{-1} B^{T} \hat{P} A+Q .
$$

The NN of the proposed system should be trained at the realtime so that the state discrepancy $\|\hat{x}(k+1)-x(k+1)\|$ becomes as small as possible at each step $k$. An energy function $E(k)$ is defined as the discrepancy between the behavior of the nominal system according to the LQR method and the one of the uncertain discrete-time system of step $k$. At each step, the weight coefficients are modified so as to minimize $E(k)$ which is given by

$$
E(k):=\frac{1}{2}(\hat{x}(k+1)-x(k+1))^{T}(\hat{x}(k+1)-x(k+1)) .
$$


If $E(k)$ can be minimized as small as possible, the discrepancy $\|\hat{x}(k+1)-x(k+1)\|^{2}$ would be also minimized so that the cost of the uncertain discrete-time system is close to the cost of the nominal system based on the LQR control.

In the learning of $\mathrm{NN}$, the modification of the weight coefficient $\Delta w_{i j}(k)$ is given by

$$
\begin{aligned}
w_{g}^{i j}(k+1) & =w_{g}^{i j}(k)+\Delta w_{g}^{i j}(k), \\
\Delta w_{g}^{i j}(k) & =-\varepsilon \frac{\partial E(k)}{\partial w_{g}^{i j}(k)}, \\
\frac{\partial E(k)}{\partial w_{g}^{i j}(k)} & =\frac{\partial E(k)}{\partial N(k)} \cdot \frac{\partial N(k)}{\partial w_{g}^{i j}(k)},
\end{aligned}
$$

where $\varepsilon$ is the learning ratio. The term $\frac{\partial E(t)}{\partial N(k)}$ can be calculated from the energy function (19) as follows:

$$
\frac{\partial E(k)}{\partial N(k)} \approx-(\hat{x}(k+1)-x(k+1)) \Gamma(k) B D_{k} E_{k},
$$

It should be noted that suppose there exists a parameter $\Gamma(k)$ such that $B+D F(k) E_{b} \approx \Gamma(k) B$, where $\Gamma(k)$ is the matrix value function and its elements are the positive scalar. Moreover, (20b) can be rewritten from (21) as follows:

$$
\Delta w_{g}^{i j}(k) \approx \eta(\hat{x}(k+1)-x(k+1)) B D_{k} E_{k} \frac{\partial N(k)}{\partial w_{g}^{i j}(k)},
$$

where $\eta:=\varepsilon\|\Gamma(k)\|$ is defined as a new learning ratio. $\eta$ is used instead of deciding $\varepsilon$ according to $\Gamma(k) \cdot \frac{\partial N(k)}{\partial w_{g}^{i j}(k)}$ can be calculated using the chain rule on the NN. From (19), (20), (21), and (22), NN can be trained so as to decrease the cost $J$ on-line.

\section{B. Multilayered Neural networks}

The utilized NN are of a three-layer feed-forward network as shown in Fig. 2. The linear function is utilized in the neurons of the input and the hidden layers, and a sigmoid function in the output layer. The inputs and outputs of each layer can be described as follows.

$$
\begin{aligned}
& s_{g}^{i}(k) \\
&:=\left\{\begin{array}{cl}
U_{i}(k) & \{g=1 \text { (input layer) }\} \\
\sum w_{1}^{(i, j)}(k) o_{1}^{j}(k) & \{g=2 \text { (hidden layer) }\} \\
\sum w_{2}^{(i, j)}(k) o_{2}^{j}(k) & \{g=3 \text { (output layer) }\},
\end{array}\right. \\
&:=\left\{\begin{array}{cc}
o_{g}^{i}(k) & \{g=1 \text { (input layer) }\} \\
s_{1}^{i}(k)+\theta_{1}^{(i)}(k) & \{g=2 \text { (hidden layer) }\} \\
\frac{1-e^{\left(-s_{3}^{i}(k)+\theta_{2}^{(i)}(k)\right)}}{1+e^{\left(-s_{3}^{i}(k)+\theta_{2}^{(i)}(k)\right)}} & \{g=3 \text { (output layer) }\},
\end{array}\right.
\end{aligned}
$$

where $s_{g}^{i}(k)$ and $o_{g}^{i}(k)$ are the input and the output of the neuron $i$ in the $g$ th layer at the step $k . w_{g}^{i, j}(k)$ indicates the weight coefficient from the neuron $j$ in the $g$ th layer to the neuron $i$ in the $(g+1)$ th layer. $U_{i}(k)$ is the input of $\mathrm{NN}$. $\theta_{g}^{(i)}(k)$ is a positive constant for the threshold of the neuron

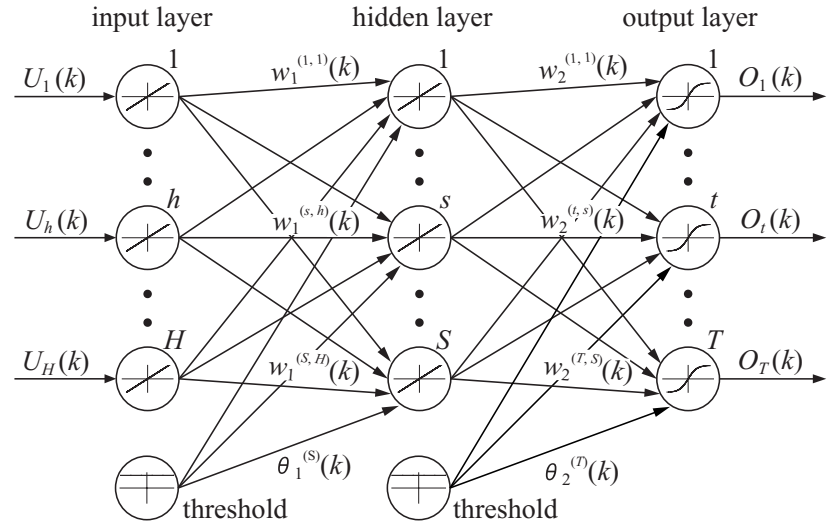

Fig. 2. Structure of the multilayered neural networks.

$i$ in the $(g+1)$ th layer. As the additive gain perturbations defined in the formula (2), the outputs of $\mathrm{NN}$ are set in the range of $[-1.0,1.0]$.

\section{CONCLUSIONS}

The application of neural networks to the guaranteed cost control problem of the discrete-time uncertain system has been investigated. Using the BMI technique, the class of the state feedback gain has been derived. The robust stability of the closed-loop system has been guaranteed by substituting the neurocontroller into the gain perturbations, even if the systems include NN. Moreover, the reduction of the cost can be attained by using neurocontroller.

\section{REFERENCES}

[1] L. Xie and Y.C. Soh, "Guaranteed cost control of uncertain discretetime systems," Control Theory and Advanced Technology, vol.10, no.4, Part 2, pp.1235-1251, 1995.

[2] J.H. Park, "Guaranteed cost control of uncertain large-scale discretetime systems," Systems Analysis Modeling Simulation, vol.43, no.2, pp.121-135, Feb. 2003.

[3] J.H. Park, "Robust non-fragile control for uncertain discrete-delay large-scale systems with a class of controller gain variations," Applied Mathematics and Computation, vol.149, iss.1, pp.147-164, Feb. 2004.

[4] K.C. Goh, M.G. Safonov and G.P. Papavas-silopoulos, "Global optimization for the biaffine matrix inequality problem,". Journal of Global Optimization, vol.7, pp.365-380, 1995.

[5] Y. Iiguni, H. Sakai and H. Tokumaru, "A nonlinear regulator design in the presence of system uncertainties using multilayered neural networks", IEEE Trans. Neural Networks, vol.2, no.4, pp.410-417, July 1991.

[6] Y. Wang, D. J. Hill and G. Guo, "Robust decentralized control for multimachine power systems," IEEE Trans. Circuits and Systems I: Fundamental Theory and Applications, vol.45, no.3, pp.271-279, Mar. 1998.

[7] K. Zhou, Essentials of Robust Control, Prentice-Hall, New Jersey, 1998

[8] H. Mukaidani, Y. Ishii, Y. Tanaka, N. Bu and T. Tsuji, "LMI based neurocontroller for guaranteed cost control of discrete-time uncertain system," Proc. 43th IEEE Conf. Decision and Control, Bahamas, Dec. 2004 (to appear).

[9] Y. Ishii, H. Mukaidani, Y. Tanaka, N. Bu and T. Tsuji, "LMI based neurocontroller for output-feedback guaranteed cost control of discretetime uncertain system," Proc. IEEE Int. Midwest Symp. Circuits and Systems, vol. III, pp.141-144, Hiroshima, July 2004. 